\title{
Relation of multi-marker panel to incident chronic kidney disease and rapid kidney function decline in African Americans: the Jackson Heart Study
}

Stanford E. Mwasongwe ${ }^{1 *}$ (D), Bessie Young ${ }^{2,3}$, Aurelian Bidulescu ${ }^{5}$, Mario Sims ${ }^{4}$, Adolfo Correa $^{4}$ and Solomon K. Musani ${ }^{4}$

\begin{abstract}
Background: Few investigations have evaluated the incremental usefulness of multiple biomarkers representing varying physiological pathways for predicting risk of renal outcomes in African Americans.

Design, setting, participants, and measurements: We related a multi-marker panel to incident chronic kidney disease (CKD) and rapid kidney function decline (RKFD) in 2813 Jackson Heart Study participants without prevalent CKD at exam 1 (2000-2004) and with complete assays at exam 1 for 9 biomarkers: adiponectin, aldosterone, B-natriuretic peptide [BNP], cortisol, high sensitivity C-reactive protein (hsCRP), endothelin, homocysteine, plasma renin activity and mass. Incident CKD was defined as estimated glomerular filtration rate (eGFR) $<60 \mathrm{~mL} / \mathrm{min} / 1$. $73 \mathrm{~m}^{2}$ at exam 3 while RKFD was defined as eGFR $\geq 30 \%$ loss between exams 1 and 3 (8.2 median years). We employed multiple logistic regression model to describe association between the panel and incident CKD and RKFD and used backward elimination strategy to estimate the most parsimonious biomarker model while controlling for conventional risk factors.
\end{abstract}

Results: The multi-marker panel predicted the risk for both incident CKD (odds ratios [OR], 2.72; 95\% confidence intervals $[\mathrm{Cl}], 1.63,4.56 ; P=0.001)$ and $\operatorname{RKFD}(2.61 ; 95 \% \mathrm{Cl}, 1.67,4.08 ; P<0.001)$. Per standard deviation increase in log biomarker concentrations were significantly (multivariable adjusted odds ratios, [95\% confidence interval], $p$-value) associated with incident CKD: plasma adiponectin $(1.24[1.07,1.44], p=0.005)$ and leptin $(1.3[1.06,1.61]$, $p=0.011)$, and with RKFD: plasma adiponectin (1.22 [1.06, 1.40], $p=0.006)$; $\operatorname{hsCRP}(1.17[1.01,1.36], p=0.031)$ and aldosterone $(0.85[0.74,0.96], p=0.012)$. Moderate levels (3rd quartile) of aldosterone were inversely associated with incident $\operatorname{CKD}(0.54[0.35,0.82], p=0.004)$ while leptin was associated with $\operatorname{RKFD}(1.64[1.10,2.44], p=0.015)$. Biomarkers improved CKD risk prediction $(P=0.003)$ but not RKFD risk prediction $(P=0.10)$.

Conclusion: In this community-based sample of African Americans, a multi-marker panel added only moderate predictive improvement compared to conventional risk factors.

Keywords: Biomarker, Chronic kidney disease, Rapid kidney function decline, Estimated glomerular filtration rate, African Americans

\footnotetext{
* Correspondence: smwasongwe@umc.edu; smwasogwe@umc.edu

'Jackson Heart Study, Jackson State University, 350 W. Woodrow Wilson Ave.,

Suite 701, Jackson, MS 39213, USA

Full list of author information is available at the end of the article
}

(c) The Author(s). 2018 Open Access This article is distributed under the terms of the Creative Commons Attribution 4.0 International License (http://creativecommons.org/licenses/by/4.0/), which permits unrestricted use, distribution, and reproduction in any medium, provided you give appropriate credit to the original author(s) and the source, provide a link to the Creative Commons license, and indicate if changes were made. The Creative Commons Public Domain Dedication waiver (http://creativecommons.org/publicdomain/zero/1.0/) applies to the data made available in this article, unless otherwise stated. 


\section{Background}

Chronic kidney disease (CKD) is a significant health problem which is associated with increased morbidity and mortality, making its prevention a public health priority. Thirteen percent of the adult population of the United States have reduced kidney function or albuminuria [1]. Early identification of persons at greater risk of developing CKD is critical in prevention and management strategies. Traditionally, hypertension and diabetes are the most commonly known key risk factors for CKD. Others include advanced age, low high-density lipoprotein cholesterol (HDL) and metabolic syndrome [2]. Previous studies have shown that traditional factors alone are inadequate to explain CKD risks and improve risk stratification for CKD or progression of CKD [3-5]. Established CKD risk factors explain only $34 \%$ of renal disease progression among whites and 44\% for African Americans after adjusting for sociodemographic, lifestyle and clinical factors $[3,6]$. In clinical settings, CKD prognosis largely depends on traditional markers such as estimated glomerular filtration rate (eGFR) and albuminuria, however these biomarkers only offer modest risk prediction particularly in people with preserved levels of renal function [7] and are subject to intra-individual variability over time when hydration and medication use are involved. Additionally, albuminuria and eGFR can have a variable relationship, an example being the development of CKD (eGFR $<60 \mathrm{~mL} / \mathrm{min} /$ $1.73 \mathrm{~m}^{2}$ ) without albuminuria [8, 9]. Several other pathways may be involved in CKD development including inflammation and endothelial function [3, 10]. Studies in cardiovascular disease (CVD) and metabolic syndrome, have benefited from the use of circulating biomarkers in risk prediction [11-13]. Unlike biomarkers in CVD, the list of prognostic biomarkers in CKD is in continuous growth and the concept of a multi-marker approach has been proposed as single biomarkers are unable to fully describe changes in renal function [14]. While multi-marker approach to predict CKD has been reported in whites [10], the predictive value of models incorporating multiple biomarkers in CKD prediction among African Americans is not well studied.

In a community-based sample of African Americans enrolled in the Jackson Heart Study (JHS), we sought to identify biomarkers of interest and evaluate their incremental predictive value from a multi-marker panel representing physiological pathways implicated in kidney diseases: adiposity (adiponectin and leptin); adrenal (aldosterone and cortisol); endothelial function (endothelin and homocysteine); inflammation $(\mathrm{C}$ - reactive protein, [CRP]); natriuretic (B-type Natriuretic Peptide [BNP]) and renin angiotensin (plasma renin activity, and renin mass). We conducted tests on model improvement using both the C-statistic and the newer measures of net reclassification index (NRI) and integrated discrimination index (IDI) $[15,16]$.

\section{Methods \\ Study sample}

The JHS is a single-site community-based prospective study designed to identify risk factors for cardiovascular disease in African Americans. The recruitment details have been summarized previously [17, 18]. Briefly, the study enrolled 5306 participants $\geq 21$ years of age (clinic Exam 1, September 2000 to March 2004) from urban and rural areas of three counties (Hinds, Madison, and Rankin) that comprises a Jackson, Mississippi Metropolitan Statistical Area (MSA). Participants were asked to return for a second clinic Exam (October 2005 to December 2008) and third clinic Exam (February 2009 to January 2013). The 9 biomarkers studied in this analysis were measured at Exam 1, while serum creatinine was measured at Exam 1 and 3 (8.2 median follow-up years). Analysis was restricted to 2813 participants after we excluded participants who (i) were missing serum creatinine values measured at Exam 1 and clinic Exam 3, $n=1548$; (ii) had prevalent CKD at baseline or reported being on dialysis, $n=202$; and (iii) were missing biomarker data, $n=743$. Data were imputed for 206 participants with missing covariates. The institutional review board at the University of Mississippi Medical Center, Jackson State University, and Tougaloo College approved the study. All participants provided informed written consent.

\section{Definition of renal outcomes, biomarker selection and measurement}

Incident CKD and RKFD, were both defined based on serum creatinine measured at Exams 1 and 3, as serum creatinine was not measured at the Exam 2. Serum creatinine was measured Exam 1 (2000-2004) and Exam 3 (2009-2013), serum creatinine was measured using a multipoint enzymatic spectrophotometric assay with the Vitros Ortho-Clinical Diagnostics Analyzer (Raritan, NJ). As part of the calibration study, measurement of serum creatinine were repeated for a random sample of 206 in 2006 using the enzymatic method on the Roche Modular P Chemistry Analyzer (Roche Diagnostics Corporation, Indianapolis, IN $[19,20]$. Serum creatinine measured at Exam 1 was then calibrated to harmonize with serum creatinine measured at Exam 3 using isotope-dilution mass spectrometry traceable method [21]. We defined both endpoints based on the change between clinic Exams 1 and 3, and eGFR was estimated using Chronic Kidney Disease Epidemiology Collaboration (CKD-EPI) creatinine equation [21-23]. Incident CKD was defined as eGFR $\geq 60 \mathrm{~mL} / \mathrm{min} / 1.73 \mathrm{~m}^{2}$ at Exam 1 (i.e., no CKD at baseline) and eGFR $<60 \mathrm{~mL} / \mathrm{min} / 1.73 \mathrm{~m}^{2}$ at Exam 3 [24]. For RKFD, the difference expressed as a percentage 
of baseline eGFR represented progression if greater than $30 \%$ [2, 25]. Any eGFR $>120 \mathrm{~mL} / \mathrm{min} / 1.73 \mathrm{~m}^{2}$ was truncated to $120 \mathrm{~mL} / \mathrm{min} / 1.73 \mathrm{~m}^{2}$ to avoid large changes in those with high normal eGFRs [26, 27]. Positive values indicate a decline of eGFR from Exam 1 to Exam 3.

Nine biomarkers were selected because of the reported associations with kidney function, biologic plausibility and availability at first examination cycle. We measured high-sensitivity C-reactive protein (a marker of inflammation); adiponectin and leptin (adiposity); aldosterone, plasma renin activity, active renin mass concentration and B-type natriuretic peptide (markers of neuro-hormonal activity); homocysteine and endothelin (markers of endothelial function and oxidant stress). A detailed description of the standard assays were used for all biomarkers with coefficient of variations reported by Musani et al. [12]. Briefly, venous blood samples were withdrawn from study participants following 8-h fasting and stored at the JHS central repository in Minneapolis, MN, USA at $-70{ }^{\circ} \mathrm{C}$ until assayed $[19,28]$.

\section{Covariate assessments}

The baseline examination included a complete medical history, physical examination and blood/urine collections. Prevalent diabetes was defined according to the American Diabetes Association (ADA) criteria as fasting ( $\geq 8 \mathrm{~h}$ ) glucose $\geq 126 \mathrm{mg} / \mathrm{dL}$, or hemoglobin A1c $(\mathrm{HbA1C}) \geq 6.5 \%$ or use of diabetic medication (actual or self-reported) within 2 weeks prior to the clinic visit). Body mass index (BMI) was defined as the weight in kilograms divided by the height in meters squared. Clinic blood pressure (BP) was measured using random zero sphygmomanometer (Hawksley and Sons Ltd., Lancing, UK) following appropriate procedure; whereby the participants' rested for 5 min in an upright position with their back and arms supported and a trained staff took two BP measurements in the right arm. The two clinic-measured BP were averaged to obtain the BP value used in the analysis. Fasting total and high-density lipoprotein (HDL) cholesterol were measured and quantified by an oxidase method and expressed as total to HDL ratio. Nephalometric immunoassay and enzymatic methods were used to quantify urinary albumin from a timed 24-h urine collection and a random spot morning urine collection [19]. Albuminuria was defined as a urinary-albumin-to-urinary-creatinine ratio (ACR) $\geq 30 \mathrm{mg} / \mathrm{g}$ using both methods. Current smoking status was defined as yes in participants who had smoked over 400 cigarettes in their lifetime and were actively smoking at the time of the baseline examination.

\section{Statistical analysis}

All biomarkers were naturally log-transformed and standardized (mean $=0$ and variance $=1$ ) within sex to normalize their skewed distributions and also to account for sex-related differences. We performed separate analyses for incident CKD and RKFD by fitting 2 different models: i) the traditional model that consisted of known independent risk factors for CKD such as systolic blood pressure, hypertension, use of antihypertensive medication, current smoking status, body mass index, total cholesterol to HDL ratio, and diabetes; and ii) the biomarker model that consisted of CKD traditional risk factors and biomarkers. For the biomarker model, we first tested the relation of the entire biomarker panel with each outcome, and if the biomarker panel was statistically significant, we used backward elimination to identify a parsimonious subset of biomarkers that remained significantly associated with incident CKD and RKFD. The retained biomarkers were thereafter used to construct weighted multi-marker score following the approach applied by Wang et al. [29]. The sum of sex-standardized log-biomarker concentration weighed by the estimated regression coefficients of each selected biomarker constituted the risk score on a continuous scale. We then used the risk score as a continuous predictor or categorized using quartiles to evaluate its association with each outcome. For secondary analyses, we stratified by obesity status in our effort to understand obesity's moderating effects on the association of biomarkers with the outcomes, considering that the Jackson Heart Study participants are on average obese (BMI > $30 \mathrm{~kg} / \mathrm{m}^{2}$ ). Additionally, we compared the biomarkers distributions between included versus excluded participants using Wilcoxon rank-sum test.

To understand the utility of the biomarkers in the prediction of incident CKD and RKFD we compared the performance of the biomarker model with the traditional model. We computed performance metrics that included change in the C-statistic to assess model discrimination, and Integrated discrimination index (IDI) and net reclassification index (NRI) $[16,30]$ to assess reclassification improvement. IDI and NRI quantifies the model's ability to predict outcome when biomarkers are included in addition to traditional risk factors. Calibration was evaluated with Hosmer-Lemeshow goodness-of-fit test with $P$-value $<0.05$ indicating a poorly calibrated model. Data were imputed for participants with missing covariates $(n=206)$, with 20 data sets using fully conditional specification (FCS) [31]. In FCS, imputations are generated sequentially by specifying an imputation model for each variable given the other variables. In this way, FCS is suited for imputing data of variables with different scales and complex relations with each other. The percentage of missing for each variable included in the analysis was $0.07 \%$ for BMI, $0.11 \%$ for SBP, $5.65 \%$ for total cholesterol to HDL ratio, $0.78 \%$ for antihypertensive medication, $0.04 \%$ for diabetes and $0.78 \%$ for current cigarette smoking 
status. We used the SAS EG statistical software version 7.1 for all statistical tests and SAS macro developed by Kennedy and Pencina for model performance evaluation [32]. All statistical significance was defined as two-tailed $P \leq 0.05$. For analysis of the structure of association between biomarkers and outcome, we used Generalized Additive Models implemented in $\mathrm{R}$ to check for non-linearity.

\section{Results}

\section{Baseline study characteristics}

Demographic, clinical and biomarker distributions at exam 1 are presented in Table 1. We included $2813 \mathrm{JHS}$ participants in our analytic cohort based on various inclusion criteria. A consort diagram of the exclusions performed in our analyses is shown in Fig. 1. Mean age of the study cohort at baseline was 54 years, $62.8 \%$ were women, the mean body mass index (BMI) was $31.9 \pm 7.3$, while $16.6 \%$ had diabetes and $47.5 \%$ reported taking blood pressure medications. The average estimated GFR at baseline was $98.08 \pm 17.76 \mathrm{ml} / \mathrm{min} / 1.73 \mathrm{~m}^{2}$. eGFR declined $9.93 \%$ (median 8.10; ranged from 0.00 to $18.00 \%$ ) between Exam 1 and Exam 3 (8.2 median follow-up years).

Table 1 Baseline characteristics of study participants $(N=2607)$

\begin{tabular}{ll}
\hline Characteristics & Mean \pm SD \\
Age, years & $53 \pm 12$ \\
Female, \% (n) & $63(1636)$ \\
BMI, kg/m & $31.9 \pm 7.3$ \\
SBP, $\mathrm{mmHg}$ & $125.9 \pm 15.7$ \\
Baseline eGFR, mL/min per 1.73 m² & $98.1 \pm 17.7$ \\
Total cholesterol to HDL ratio & $4.1 \pm 1.3$ \\
Blood pressure medications, \% (n) & $47.5(1238)$ \\
Diabetes, \% (n) & $16.6(432)$ \\
Current smoking, \% (n) & $11.7(306)$ \\
Biomarker level & Median $(25$ th, 75th percentiles) \\
Adiponectin, ng/mL & $4037.0(2640.1,6339.2)$ \\
Aldosterone, $\mathrm{ng} / \mathrm{mL}$ & $4.3(2.5,6.9)$ \\
BNP, pg/mL & $6.7(2.3,14.8)$ \\
hsCRP, mg/dL & $0.3(0.1,0.6)$ \\
Endothelin, pg/mL & $1.2(0.9,1.6)$ \\
Homocysteine, $\mu \mathrm{mol} / \mathrm{dL}$ & $8.4(7.2,9.9)$ \\
Leptin, $\mathrm{ng} / \mathrm{mL}$ & $22.9(10.1,39.3)$ \\
Plasma renin activity, ng/mL/hr & $0.4(0.2,1.0)$ \\
Active renin mass concentration, pg/mL & $6.7(5.1,9.4)$ \\
\hline
\end{tabular}

Data presented as mean \pm standard deviation (SD) for continuous variables and percentage (count) for dichotomous variables unless otherwise indicated

Abbreviations: $B M I$ body mass index, SBP systolic blood pressure, eGFR estimated glomerular filtration rate, $H D L$ high-density lipoprotein cholesterol, hsCRP high-sensitive c-reactive protein, BNP B-type natriuretic peptide

\section{Association of multi-marker panel with incident CKD}

During a median follow-up period of 8.2 years, $10.5 \%$ ( $n=178$ women) of participants developed incident CKD. We observed that the multi-marker panel was significantly associated with the development of CKD $(P=0.004)$ on follow-up. Upon backward elimination, continuous $\log$ plasma adiponectin $(P=0.005)$ and leptin concentrations $(P=0.011)$ were retained as significantly associated with incident CKD. We also tested the association of high levels (based on data derived quartiles) of plasma adiponectin and leptin with incident CKD. The multivariable adjusted odds ratios (ORs) and 95\% confidence intervals (95\% CI) are summarized in Table 2. High levels of log plasma adiponectin (OR, 1.66; 95\% CI, 1.08-2.55) and leptin (OR, 2.00; 95\% CI 2.00; $P$-value $=0.022) ; 95 \%$ CI, $1.18-3.38 ; \quad P$-value $=0.009)$ were significantly associated with incident CKD. In addition, moderate levels (second quartile) of plasma adiponectin were also significantly associated with incident CKD. When we combined adiponectin and leptin to form a multi-marker score, and found that the ORs almost doubled for the continuous multi-marker score. Both high and moderate levels of the multi-marker score were significantly associated with incident CKD. In Fig. 2a, the smoother splines show the structure of the multivariable relationship between plasma aldosterone with CKD. Moderate level of plasma aldosterone appeared to be protective against incident CKD development.

\section{Association of multi-marker panel with rapid kidney function decline}

During a median follow-up period of 8.2 years, $11.0 \%$ ( $n=202$ women) of participants developed RKFD, and the multi-marker panel was significantly associated with RKFD ( $p$-value $=0.001)$. Adiponectin and aldosterone were retained as significant correlates of RKFD (Table 2). When divided into quartiles, with exception of aldosterone, which was protective (OR, 0.62; 95\% CI, 0.43-0.89; $P$-value $=0.009$ ), the highest quartile for adiponectin (OR, 1.49; 95\% CI, 1.10-2.44; $P$-value $=0.045)$ and medium quartile leptin level (OR, 1.64; 95\% CI, $1.10-2.44 ; P$-value $=0.015)$ were not significantly associated with RKFD. A multi-marker score that comprise the three biomarkers was significantly associated with the risk for RKFD both as a continuous and categorical states. A smoother splines, Fig. 2b depict the multivariable relationship between leptin with RKFD.

\section{Secondary analyses}

For secondary analyses, we compared the biomarkers distributions of participants included versus those 


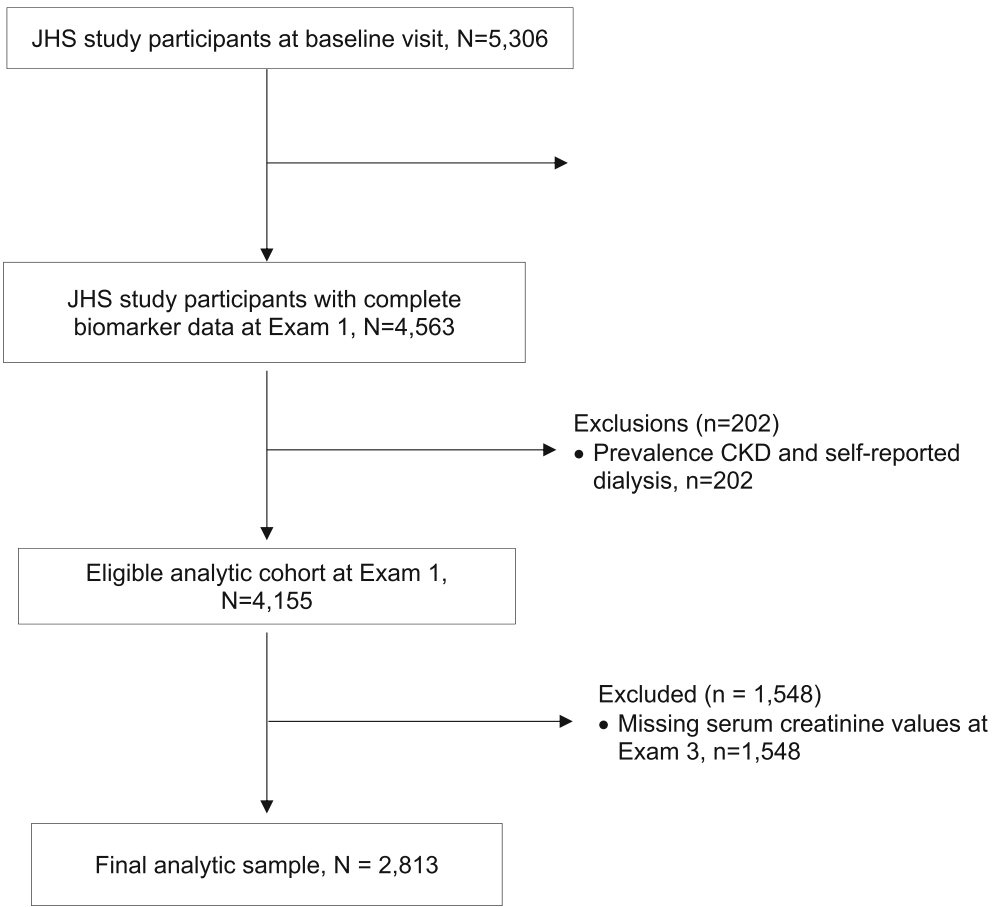

Fig. 1 CONSORT flow diagram for the relation of multi-marker panel and incident chronic kidney disease (CKD) and rapid kidney function decline (RKFD)

excluded from the analyses due and results are summarized on Additional file 1: Table S1. We also repeated the association analyses stratified by obesity status to assess whether obesity moderates the biomarker-incident CKD / RKFD relation. Only adiponectin concentrations interacted significantly with obesity status $(P$-value $=0.016)$. Results of the analysis stratified by obesity status, showed that high levels of both adiponectin and medium level of leptin were significantly associated with development of CKD among non-obese participants but not among obese participants. Similar results were evident for the multi-marker score combining adiponectin and leptin although among obese participants, the highest quartile (4th) of the risk score was also significantly associated with CKD. Stratified analyses of the association of adiponectin, aldosterone and leptin with RKFD showed a similar pattern as for incident CKD. In the leptin-RKFD relation however, the second quartile was significantly associated with RKFD but not the other quartiles suggesting possible non-linear relation (Additional file 1: Table S3).

\section{Evaluation of model performance}

The added predictive ability of the biomarker model in terms of improved discrimination and reclassification above the conventional CKD risk factors is shown in Table 3. The biomarker model exceeds the traditional CKD risk factor model by $1 \%$ in predicting incident
CKD and RKFD. With respect to reclassification improvement, the biomarker model reclassified $11 \%$ and $15 \%$ CKD and RKFD events, respectively compared to the traditional model. Moreover, the predicted mean probability of events was significantly different between biomarker and traditional CKD risk factor models, with the former performing better than the later.

\section{Discussion}

We investigated the relation of a multi-marker panel with the development of incident CKD and RKFD in a large community-based sample of African Americans. We observed that a panel consisting of nine circulating biomarkers (adiponectin, aldosterone, BNP, hsCRP, endothelin, homocysteine, leptin, PRA, ARM) representing several distinct biologic pathways was associated with development of CKD and RKFD. We identified a smaller subset of biomarkers representing adiposity (adiponectin, leptin); and RAS (aldosterone) pathways that were also associated with these outcomes. Plasma adiponectin and leptin were both associated with development of CKD while plasma aldosterone had a protective effects against both CKD development and RKFD. The addition of biomarkers only marginally improved model discrimination and reclassification compared to the model with traditional risk factors as demonstrated by the small change in C-statistic and reclassification indices. In secondary analyses stratified by obesity status, selected biomarkers were significantly associated with 
Table 2 Associations of multi-marker panel, individuals' biomarkers and multi-marker scores with incident CKD and rapid kidney function decline

\begin{tabular}{|c|c|c|c|c|c|c|}
\hline \multirow[t]{2}{*}{ Biomarkers } & \multicolumn{3}{|l|}{ Incident CKD } & \multicolumn{3}{|c|}{ Rapid Kidney Function Decline (RKFD) } \\
\hline & Cases/ \# at risk & $\begin{array}{l}\text { Multivariable Adjusted } \\
\text { Odds Ratio }(95 \% \mathrm{Cl})\end{array}$ & $P$-value & Cases/\# at risk & $\begin{array}{l}\text { Multivariable Adjusted } \\
\text { Odds Ratio }(95 \% \mathrm{Cl})\end{array}$ & $P$-value \\
\hline Entire panel & & $2.72(1.63,4.56)$ & 0.001 & & $2.61(1.67,4.08)$ & 0.001 \\
\hline \multicolumn{7}{|l|}{ Adiponectin } \\
\hline Continuous & & $1.24(1.07,1.44)$ & 0.005 & & $1.22(1.06,1.40)$ & 0.006 \\
\hline Q1 & $54 / 703$ & Reference & & $63 / 703$ & Reference & \\
\hline Q2 & $71 / 703$ & $1.37(0.89,2.09)$ & 0.151 & 78/703 & $1.20(0.83,1.75)$ & 0.330 \\
\hline Q3 & $72 / 704$ & $1.31(0.85,2.02)$ & 0.217 & $72 / 704$ & $1.14(0.77,1.67)$ & 0.515 \\
\hline Q4 & $98 / 703$ & $1.66(1.08,2.55)$ & 0.022 & $97 / 703$ & $1.49(1.02,2.18)$ & 0.045 \\
\hline \multicolumn{7}{|l|}{ Leptin } \\
\hline Continuous & & $1.31(1.06,1.61)$ & 0.011 & & $1.12(0.93,1.34)$ & 0.234 \\
\hline Q1 & $54 / 698$ & Reference & $\ldots$ & $67 / 698$ & Reference & $\ldots$ \\
\hline Q2 & 79/711 & $1.37(0.89,2.11)$ & 0.151 & $77 / 711$ & $1.13(0.77,1.66)$ & 0.525 \\
\hline Q3 & 73/699 & $1.52(0.95,2.42)$ & 0.079 & $90 / 699$ & $1.64(1.10,2.44)$ & 0.015 \\
\hline Q4 & $89 / 705$ & $2.00(1.18,3.38)$ & 0.009 & $76 / 705$ & $1.29(0.80,2.06)$ & 0.295 \\
\hline \multicolumn{7}{|l|}{ hsCRP } \\
\hline Continuous & & $1.13(0.96,1.33)$ & 0.149 & & $1.17(1.01,1.36)$ & 0.031 \\
\hline Q1 & $53 / 700$ & Reference & $\ldots$ & $56 / 700$ & Reference & $\ldots$ \\
\hline Q2 & $88 / 707$ & $1.22(0.80,1.87)$ & 0.358 & $81 / 707$ & $1.12(0.76,1.65)$ & 0.572 \\
\hline Q3 & $85 / 705$ & $1.32(0.86,2.02)$ & 0.212 & $88 / 705$ & $1.25(0.85,1.84)$ & 0.258 \\
\hline Q4 & $69 / 701$ & $1.13(0.71,1.80)$ & 0.593 & $85 / 701$ & $1.28(0.85,1.92)$ & 0.230 \\
\hline \multicolumn{7}{|l|}{ Aldosterone } \\
\hline Continuous & & $0.92(0.8,1.06)$ & 0.229 & & $0.85(0.74,0.96)$ & 0.012 \\
\hline Q1 & $77 / 698$ & Reference & $\ldots$ & $93 / 698$ & Reference & $\ldots$ \\
\hline Q2 & $68 / 720$ & $0.82(0.55,1.23)$ & 0.347 & $80 / 720$ & $0.88(0.62,1.24)$ & 0.461 \\
\hline Q3 & $58 / 707$ & $0.54(0.35,0.82)$ & 0.004 & $66 / 707$ & $0.66(0.46,0.95)$ & 0.026 \\
\hline Q4 & $92 / 688$ & $0.68(0.46,1.04)$ & 0.077 & $71 / 688$ & $0.62(0.43,0.89)$ & 0.009 \\
\hline \multicolumn{7}{|c|}{ Multi-marker Score } \\
\hline 0 & $40 / 651$ & Reference & & $42 / 651$ & Reference & \\
\hline 1 & $53 / 652$ & $1.48(0.94-2.33)$ & 0.093 & $54 / 652$ & $1.12(0.75-1.67)$ & 0.586 \\
\hline 2 & $73 / 652$ & $2.02(1.30-3.14)$ & 0.002 & $74 / 652$ & $1.62(1.11-2.37)$ & 0.001 \\
\hline 3 & $90 / 652$ & $2.45(1.53-3.91)$ & $<.001$ & $95 / 652$ & $2.04(1.40-2.99)$ & $<.001$ \\
\hline
\end{tabular}

Abbreviations: Q1 quartile 1, Q2 quartile 2, Q3 quartile 3, Q4 quartile 4, $h s C R P$ high-sensitive C-reactive protein

Incident chronic kidney disease (CKD) was defined a decline from eGFR $\geq 60 \mathrm{~mL} / \mathrm{min} / 1.73 \mathrm{~m}^{2}$ at exam 1 to eGFR $<60 \mathrm{~mL} / \mathrm{min} / 1.73 \mathrm{~m}{ }^{2}$ at exam $3 \mathrm{follow}$-up (median follow-up duration: 8.0 years)

Rapid kidney function decline (RKFD) was defined as a decline in estimated glomerular filtration rate (eGFR) $\geq 30 \%$ from exam 1 to exam 3 (median follow-up duration: 8.0 years)

Multivariate model for the estimation of ORs were for adjusted for age, sex, baseline estimated glomerular filtration rate (eGFR), systolic blood pressure,

hypertension, use of hypertension medication, smoking, body mass index (BMI), total cholesterol to high-density lipoprotein cholesterol (HDL) ratio and diabetes

incident CKD and RKFD in non-obese participants only, suggesting modification by obesity status.

In our study, adiponectin and leptin, two of the key cytokines secreted by adipocytes, predicted the development of incident CKD in a multivariable adjusted model. These findings are consistent with previous studies that investigated their association with CKD. In a case-control study among Chinese and Indian adults, patients with CKD had higher levels of leptin and adiponectin compared to controls [33]. Similar findings were also found in a patient population comprised of $60 \%$ African Americans in the greater New Orleans, Louisiana region, after adjusting for race and other risk factors associated with kidney disease [34]. To the contrary, other studies have also reported no difference in adiponectin levels [35-37]. The link between adipokines and changes in glomerular filtration rate has 

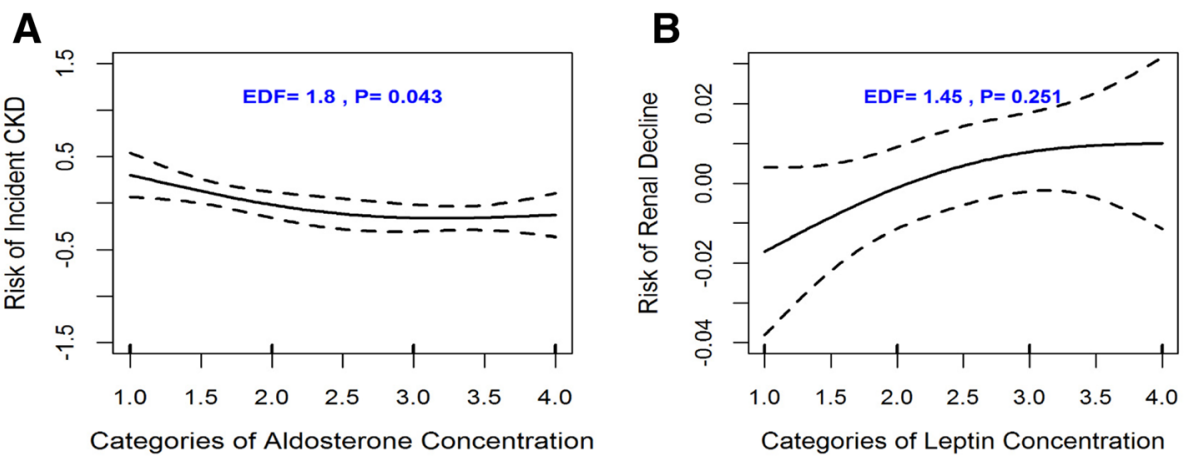

Fig. 2 Penalized spline smoother of the relationship between the risk of incident chronic kidney disease (CKD) and aldosterone (a), and between RKFD and leptin (b)

been reported previously [38]. Through endothelial dysfunction, oxidative stress and changes in immune response and inflammation, the adipokines are involved in kidney damage [39]. While serum aldosterone was reported to have weak but significant association with lower eGFR in Framingham Offspring Study, an inverse association where aldosterone appear to be protective was observed in this present study. Aldosterone's conflicting results are also reported in the Ohasama Study where the authors attributed the lack of association of aldosterone with eGFR to a high salt-intake resulting from high sodium dietary conditions $[40,41]$.

Studies on single biomarkers have reported on the relation of CRP and aldosterone with kidney function. Works by Fox and colleagues as well as Shankar and others showed that CRP is associated with prevalent CKD but not with the development of CKD [42, 43]. While previous studies both clinical and observational have demonstrated CRP's pathogenic role in renal damage $[44,45]$, in the current analysis, CRP was not associated with the development of either CKD or RKFD. Hannemann and colleagues found an inverse association of plasma aldosterone concentration with eGFR in the general population [46]. In the present study, participants with medium level quartile and higher aldosterone level had 46\% $(P=0.004)$ and 38\% $(P$-value $=0.009)$ less likely to develop CKD or experience RKFD, respectively. When stratified by obesity status, biomarkers were associated with development of CKD and RKFD in non-obese, particularly for leptin and adiponectin. Biomarkers linkages to the development of CKD in the absence of obesity has been reported before even though the mechanism is poorly understood $[38,47]$.

Few community-based studies have evaluated kidney disease biomarkers to assess their usefulness in stratifying disease risk [3]. We undertook this study to address this gap in CKD literature. Data from the Framingham Heart Study (FHS) followed a multi-marker approach to

Table 3 Incremental predictive utility of biomarkers for incident chronic kidney disease (CKD), rapid kidney function decline (RKFD) showing C-statistics and reclassification metrics

\begin{tabular}{|c|c|c|c|c|c|c|}
\hline & \multirow[t]{2}{*}{ C-Statistics } & \multicolumn{2}{|l|}{ NRI } & \multicolumn{2}{|l|}{$\mid \mathrm{DI}$} & \multirow[t]{2}{*}{ Calibration Statistics $^{\mathrm{a}}\left(X^{2}, P\right)$} \\
\hline & & $\begin{array}{l}\text { Events correctly } \\
\text { reclassified }\end{array}$ & $\begin{array}{l}\text { Non-Events correctly } \\
\text { reclassified }\end{array}$ & $\begin{array}{l}\text { Mean probability } \\
\text { for events }\end{array}$ & $\begin{array}{l}\text { Mean probability } \\
\text { for non-events }\end{array}$ & \\
\hline \multicolumn{7}{|l|}{ Chronic kidney disease (CKD) } \\
\hline Model 1: age-sex-MV & 0.87 & $11 \%$ & $5 \%$ & $32 \%$ & $7.4 \%$ & $12.93(P=0.11)$ \\
\hline Model 2: age-sex-MV-Biomarker ${ }^{\mathrm{C}}$ & 0.88 & & & $33 \%$ & $7.3 \%$ & $14.26(P=0.08)$ \\
\hline P-value comparing models 1 vs. 2 & 0.003 & 0.08 & 0.01 & 0.01 & & \\
\hline \multicolumn{7}{|l|}{ Rapid kidney function decline } \\
\hline Model 1: age-sex-MV & 0.76 & $15 \%$ & $11 \%$ & $19.3 \%$ & $9 \%$ & $12.19(P=0.14)$ \\
\hline Model 2: age-sex-MV-Biomarker ${ }^{d}$ & 0.77 & & & $20.3 \%$ & $9 \%$ & $18.42(P=0.02)$ \\
\hline P-value comparing models 1 vs. 2 & 0.10 & 0.01 & $<.0001$ & 0.0001 & & \\
\hline
\end{tabular}

Abbreviations: $N R I$ net reclassification index, IDI integrated discrimination index

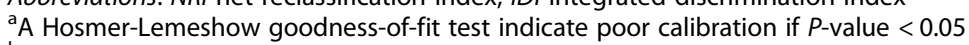

${ }^{\mathrm{b}} \mathrm{MV}$ adjusted for age, sex, baseline estimated glomerular filtration rate (eGFR), systolic blood pressure, hypertension, use of hypertension

medication, smoking, body mass index (BMI), total cholesterol to high-density lipoprotein cholesterol (HDL) ratio and diabetes

${ }^{C}$ In backward elimination of the biomarker panel, adiponectin and leptin are significant

${ }^{d}$ In backward elimination of the biomarker panel, adiponectin, high-sensitive C - reactive protein (CRP) and aldosterone are significant 
predict incident CKD and microalbuminuria. A panel of seven biomarkers (C-reactive protein, aldosterone, renin, BNP, plasminogen-activator inhibitor type 1, fibrinogen, and homocysteine) was associated with the development of CKD with homocysteine and aldosterone retained as significant markers in the backward elimination model [10]. Our data extends these findings to a large community-based sample of African Americans in Mississippi. Unlike FHS where homocysteine and aldosterone were retained as significant markers for incident CKD prediction, in JHS adiponectin and leptin were the significant markers.

When comparing indices of model improvement the biomarker model was associated with the same change in C-statistic $(\Delta C=0.01)$ for prediction of incident CKD and RKFD. Researchers generally consider a change in C-statistic of at least 0.05 as indicative of a predictor with clinical significance [48]. While $\mathrm{C}$ statistics has been criticized for being insensitive to small changes in predictive accuracy [49], it was preferred here to permit easy comparison with findings in the literature, which often used the metric [50]. We also computed the NRI and IDI indices to complement C-statistic. Consistent with results based on C-statistics for the biomarker model, relative IDI had small but significant incremental predictive ability that was also higher than that reported in FHS. NRI though statistically non-significant was higher than that reported in FHS (JHS NRI $=16.1 \%, P=0.08$; FHS NRI $=$ $6.9 \%, P=0.0004)$. Though the metrics of model improvement are study/cohort specific, suffice it to say that they hold promise for CKD prediction in African Americans as is in white populations. The utility of biomarkers in improving disease prediction is highly successful in the area of cardiovascular medicine [50-52]; however, the yield has been relatively small in women and the elderly [53, 54]. With exception of Velagaleti et al. report on prediction of heart failure $(\Delta C=0.02)$ [55], most CVD research has reported lower incremental benefit compared to current analyses. This may be because CVD risk factors are well characterized and the existence of multiple risk-algorithms aid prediction, something which is lacking in CKD research.

\section{Strengths and limitations}

This study has some strengths and limitations. The analyses had a large sample size and a well-documented spectrum of biomarkers. We also adjusted for many CKD factors so the independent association between multi-marker panel and CKD development could be assessed. Some limitations require mentioning. Our sample was primarily African Americans, limiting generalizability to other ethnicities. JHS was designed to investigate CVD risk factors, thus the biomarkers collected were not specifically for CKD, although CVD is a potent risk factor for CKD and CKD progression. Finally, with the study being observational in nature, it is possible that the CKD being detected might have developed at an earlier date.

\section{Conclusions}

In summary, while the predictive utility of biomarkers in these data were minimal, they do not exclude the role of using circulating biomarkers to provide insight into early development of CKD in this vulnerable population. Circulating adipokines (adiponectin, leptin), CRP and aldosterone biomarkers incrementally predicted incident CKD and RKFD in our large community-based sample.

\section{Additional file}

Additional file 1: Table S1. Comparing characteristics of the Included versus Excluded Participants. Table S2. Baseline characteristics and biomarkers distribution by incident chronic kidney disease (CKD) and rapid kidney function decline (RKFD). Table S3. Associations between biomarkers with incident CKD and RKFD stratified by obesity status. (DOCX $39 \mathrm{~kb}$ )

\section{Abbreviations}

ACR: Albumin-creatinine ratio; ADA: American Diabetes Association; BMI: Body mass index; BNP: B-natriuretic peptide; BP: Blood pressure; CKD: Chronic kidney disease; CKD-EPI: Chronic Kidney Disease Epidemiology Collaboration creatinine equation; CVD: Cardiovascular disease;

eGFR: Estimated glomerular filtration rate; FCS: Fully conditional specification; HDL: High-density lipoprotein; hsCRP: High sensitive C-reactive protein; IDI: Integrated discrimination index; JHS: Jackson Heart Study; NRI: Net reclassification index; RKFD: Rapid kidney function decline

\section{Acknowledgements}

The authors thank the participants and data collection staff of the Jackson Heart Study.

\section{Funding}

The Jackson Heart Study is supported by contracts HHSN268201300046C, HHSN268201300047C, HHSN268201300048C, HHSN268201300049C, HHSN268201300050C from the NHLBI and the NIMHD. This work was also supported by NIDDK grants R01 DK102134 (B.A.Y.) and R01HL117323 (P.M.). M.S. receives support through grants P60MD002249 and U54MD008176 from the NIMHD; 15SFDRN26140001 and P5OHL120163 from the American Heart

Association; and 1R01HL116446 from the NHLBI. B.A.Y. is also supported in part by funding from Veterans Affairs Puget Sound Health Care System. The funding sources have no role in the design and conduct of the study; collection, management, analysis, and interpretation of the data; preparation, review, or approval of the manuscript; or decision to submit the manuscript for publication.

\section{Disclaimer}

The views expressed in this manuscript are those of the authors and do not necessarily represent the views of the National Heart, Lung, and Blood Institute; the National Institutes of Health; or the U.S. Department of Health and Human Services.

\section{Availability of data and materials}

JHS data are available on dbGap and/or direct request addressed to the JHS leadership.

\section{Authors' contributions}

SEM interpreted and drafted the manuscript. BY, AB, MS, and AC made substantial contributions to the interpretation of the data and critical revision of the manuscript. SKM was responsible for the study conception and design, data 
analysis, interpretation of the data and drafting and critical revision of the manuscript. All authors read and approved the final manuscript.

\section{Ethics approval and consent to participate}

JHS is a population-based cohort study recruited participants from three counties (Hinds, Madison, and Rankin) that make the Jackson Metropolitan Statistical Area (MSA), Jackson, MS. Study protocols and consent forms for JHS were approved by the responsible research ethics committees and institutional review boards. All JHS participants provided written informed consent.

\section{Consent for publication}

Not applicable - all reported data have been aggregated. This manuscript does not provide participant level data.

\section{Competing interests}

The authors declare that other than the disclosures and funding sources described herein, they have no competing interests.

The views expressed in this manuscript are those of the authors and do not necessarily represent the views of the National Heart, Lung, and Blood Institute; the National Institutes of Health; the U.S. Department of Health and Human Services. The US Department of Veteran Affairs does not endorse any of the statement or opinions advocated by this article.

\section{Publisher's Note}

Springer Nature remains neutral with regard to jurisdictional claims in published maps and institutional affiliations.

\section{Author details}

'Jackson Heart Study, Jackson State University, 350 W. Woodrow Wilson Ave., Suite 701, Jackson, MS 39213, USA. ²Division of Nephrology, Kidney Research Institute University of Washington, Seattle, WA, USA. ${ }^{3}$ Veterans Affairs Puget Sound Health Care System, Seattle, WA, USA. ${ }^{4}$ Department of Medicine, University of Mississippi Medical Center, Jackson, MS, USA. ${ }^{5}$ Department of Epidemiology and Biostatistics, School of Public Health, Indiana University, Bloomington, IN, USA.

\section{Received: 6 March 2018 Accepted: 30 August 2018}

\section{Published online: 20 September 2018}

\section{References}

1. Coresh J, Selvin E, Stevens LA, Manzi J, Kusek JW, Eggers P, Van Lente F, Levey AS. Prevalence of chronic kidney disease in the United States. JAMA. 2007;298(17):2038-47.

2. Young BA, Katz R, Boulware LE, Kestenbaum B, de Boer $\mathbb{H}$, Wang W, Fulop T, Bansal N, Robinson-Cohen C, Griswold M, et al. Risk factors for rapid kidney function decline among African Americans: the Jackson Heart Study (JHS). Am J Kidney Dis. 2016;68(2):229-39.

3. Zoccali C. Biomarkers in chronic kidney disease: utility and issues towards better understanding. Curr Opin Nephrol Hypertens. 2005;14(6):532-7.

4. Peralta CA, Katz R, Sarnak MJ, Ix J, Fried LF, De Boer I, Palmas W, Siscovick D, Levey AS, Shlipak MG. Cystatin C identifies chronic kidney disease patients at higher risk for complications. J Am Soc Nephrol. 2011;22(1):147-55.

5. Weekley CC, Peralta CA. Advances in the use of multimarker panels for renal risk stratification. Curr Opin Nephrol Hypertens. 2012;21(3):301-8.

6. Tarver-Carr ME, Powe NR, Eberhardt MS, LaVeist TA, Kington RS, Coresh J, Brancati FL. Excess risk of chronic kidney disease among African-American versus white subjects in the United States: a population-based study of potential explanatory factors. J Am Soc Nephrol. 2002;13(9):2363-70.

7. Dunkler D, Gao P, Lee SF, Heinze G, Clase CM, Tobe S, Teo KK, Gerstein H, Mann JF, Oberbauer R. Risk prediction for early CKD in type 2 diabetes. Clin J Am Soc Nephrol. 2015:10(8):1371-9.

8. Peters KE, Davis WA, Ito J, Winfield K, Stoll T, Bringans SD, Lipscombe RJ, Davis TME. Identification of novel circulating biomarkers predicting rapid decline in renal function in type 2 diabetes: the Fremantle diabetes study phase II. Diabetes Care. 2017:40(11):1548-55.

9. Looker HC, Colombo M, Hess S, Brosnan MJ, Farran B, Dalton RN, Wong MC, Turner C, Palmer CN, Nogoceke E, et al. Biomarkers of rapid chronic kidney disease progression in type 2 diabetes. Kidney Int. 2015;88(4):888-96.
10. Fox CS, Gona P, Larson MG, Selhub J, Tofler G, Hwang SJ, Meigs JB, Levy D, Wang TJ, Jacques PF, et al. A multi-marker approach to predict incident CKD and microalbuminuria. J Am Soc Nephrol. 2010;21(12):2143-9.

11. Gaggin HK, Januzzi JL Jr. Biomarkers and diagnostics in heart failure. Biochim Biophys Acta. 2013;1832(12):2442-50.

12. Musani SK, Vasan RS, Bidulescu A, Liu J, Xanthakis V, Sims M, Gawalapu RK, Samdarshi TE, Steffes M, Taylor HA, et al. Aldosterone, C-reactive protein, and plasma B-type natriuretic peptide are associated with the development of metabolic syndrome and longitudinal changes in metabolic syndrome components: findings from the Jackson Heart Study. Diabetes Care. 2013; 36(10):3084-92

13. Ingelsson E, Pencina MJ, Tofler GH, Benjamin EJ, Lanier KJ, Jacques PF, Fox CS, Meigs JB, Levy D, Larson MG, et al. Multimarker approach to evaluate the incidence of the metabolic syndrome and longitudinal changes in metabolic risk factors: the Framingham offspring study. Circulation. 2007;1 16(9):984-92.

14. Mischak H, Delles C, Vlahou A, Vanholder R. Proteomic biomarkers in kidney disease: issues in development and implementation. Nat Rev Nephrol. 2015; 11(4):221-32.

15. Leening MJ, Vedder MM, Witteman JC, Pencina MJ, Steyerberg EW. Net reclassification improvement: computation, interpretation, and controversies: a literature review and clinician's guide. Ann Intern Med. 2014;160(2):122-131.

16. Pencina MJ, D'Agostino RB Sr, Steyerberg EW. Extensions of net reclassification improvement calculations to measure usefulness of new biomarkers. Stat Med. 2011;30(1):11-21.

17. Fuqua SR, Wyatt SB, Andrew ME, Sarpong DF, Henderson FR, Cunningham MF, Taylor HA Jr. Recruiting African-American research participation in the Jackson Heart Study: methods, response rates, and sample description. Ethn Dis. 2005;15(4 Suppl 6):S6-18-29.

18. Sempos CT, Bild DE, Manolio TA. Overview of the Jackson Heart Study: a study of cardiovascular diseases in african american men and women. Am J Med Sci. 1999;317(3):142-46.

19. Carpenter MA, Crow R, Steffes M, Rock W, Heilbraun J, Evans G, Skelton T, Jensen R, Sarpong D. Laboratory, reading center, and coordinating center data management methods in the Jackson Heart Study. Am J Med Sci. 2004;328(3):131-44.

20. Rebholz CM, Harman JL, Grams ME, Correa A, Shimbo D, Coresh J, Young BA. Association between endothelin-1 levels and kidney disease among blacks. J Am Soc Nephrol. 2017;28(11):3337-44.

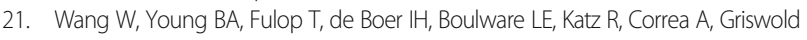
ME. Effects of serum creatinine calibration on estimated renal function in african americans: the Jackson Heart Study. Am J Med Sci. 2015;349(5):379-84.

22. Inker LA, Schmid CH, Tighiouart H, Eckfeldt JH, Feldman HI, Greene $T$ Kusek JW, Manzi J, Van Lente F, Zhang YL, et al. Estimating glomerular filtration rate from serum creatinine and cystatin C. N Engl J Med. 2012:367(1):20-9.

23. Shlipak MG, Katz R, Kestenbaum B, Siscovick D, Fried L, Newman A, Rifkin D, Sarnak MJ. Rapid decline of kidney function increases cardiovascular risk in the elderly. J Am Soc Nephrol. 2009;20(12):2625-30.

24. Bash LD, Coresh J, Kottgen A, Parekh RS, Fulop T, Wang Y, Astor BC Defining incident chronic kidney disease in the research setting: the ARIC study. Am J Epidemiol. 2009;170(4):414-24.

25. Coresh J, Turin TC, Matsushita K, Sang Y, Ballew SH, Appel L, Arima H, Chadban SJ, Cirillo M, Djurdjev O, et al. Decline in estimated glomerular filtration rate and subsequent risk of end-stage renal disease and mortality. Jama. 2014:311(24):2518-31.

26. Stevens LA, Schmid CH, Greene T, Zhang YL, Beck GJ, Froissart M, Hamm LL, Lewis JB, Mauer M, Navis GJ, et al. Comparative performance of the CKD epidemiology collaboration (CKD-EPI) and the modification of diet in renal disease (MDRD) study equations for estimating GFR levels above $60 \mathrm{~mL} /$ min/1.73 m2. Am J Kidney Dis. 2010;56(3):486-95.

27. Hiramoto JS, Katz R, Peralta CA, Ix JH, Fried L, Cushman M, Siscovick D, Palmas W, Sarnak M, Shlipak MG. Inflammation and coagulation markers and kidney function decline: the multi-ethnic study of atherosclerosis (MESA). Am J Kidney Dis. 2012;60(2):225-32.

28. Taylor HA Jr, Wilson JG, Jones DW, Sarpong DF, Srinivasan A, Garrison RJ, Nelson C, Wyatt SB. Toward resolution of cardiovascular health disparities in African Americans: design and methods of the Jackson Heart Study. Ethn Dis. 2005:15(4 Suppl 6):S6-4. 17

29. Wang TJ, Larson MG, Levy D, Benjamin EJ, Leip EP, Omland T, Wolf PA, Vasan RS. Plasma natriuretic peptide levels and the risk of cardiovascular events and death. N Engl J Med. 2004;350(7):655-63. 
30. Pencina MJ, D'Agostino RB Sr, D'Agostino RB Jr, Vasan RS. Evaluating the added predictive ability of a new marker: from area under the ROC curve to reclassification and beyond. Stat Med. 2008;27(2):157-72. discussion 207-112

31. Liu Y, De A. Multiple imputation by fully conditional specification for dealing with missing data in a large epidemiologic study. Int J Stat Med Res. 2015;4(3):287-95.

32. Kennedy K, Pencina M: A SAS ${ }^{\circledR}$ macro to compute added predictive ability of new markers predicting a dichotomous outcome. SouthEeast SAS users group annual meeting proceedings 2010.

33. Lim CC, Teo BW, Tai ES, Lim SC, Chan CM, Sethi S, Wong TY, Sabanayagam C. Elevated serum leptin, adiponectin and leptin to adiponectin ratio is associated with chronic kidney disease in Asian adults. PLoS One. 2015; 10(3):e0122009.

34. Mills KT, Hamm LL, Alper AB, Miller C, Hudaihed A, Balamuthusamy S, Chen CS, Liu Y, Tarsia J, Rifai N, et al. Circulating adipocytokines and chronic kidney disease. PLoS One. 2013;8(10):e76902.

35. Guebre-Egziabher F, Bernhard J, Funahashi T, Hadj-Aissa A, Fouque D. Adiponectin in chronic kidney disease is related more to metabolic disturbances than to decline in renal function. Nephrol Dial Transplant. 2005;20(1):129-34.

36. Yaturu S, Reddy RD, Rains J, Jain SK. Plasma and urine levels of resistin and adiponectin in chronic kidney disease. Cytokine. 2007;37(1):1-5.

37. Becker B, Kronenberg F, Kielstein JT, Haller H, Morath C, Ritz E, Fliser D. Renal insulin resistance syndrome, adiponectin and cardiovascular events in patients with kidney disease: the mild and moderate kidney disease study. J Am Soc Nephrol. 2005;16(4):1091-8.

38. Briffa JF, McAinch AJ, Poronnik P, Hryciw DH. Adipokines as a link between obesity and chronic kidney disease. Am J Physiol Renal Physiol. 2013; 305(12):F1629-36.

39. Ruster C, Wolf G. Adipokines promote chronic kidney disease. Nephrol Dial Transplant. 2013;28(Suppl 4):iv8-14.

40. Terata S, Kikuya M, Satoh M, Ohkubo T, Hashimoto T, Hara A, Hirose T, Obara T, Metoki H, Inoue $\mathrm{R}$, et al. Plasma renin activity and the aldosteroneto-renin ratio are associated with the development of chronic kidney disease: the Ohasama study. J Hypertens. 2012;30(8):1632-8.

41. Jurgens $\mathrm{G}$, Graudal NA. Effects of low sodium diet versus high sodium diet on blood pressure, renin, aldosterone, catecholamines, cholesterols, and triglyceride. Cochrane Database Syst Rev. 2004;(1):Cd004022.

42. Fox ER, Benjamin EJ, Sarpong DF, Nagarajarao H, Taylor JK, Steffes MW, Salahudeen AK, Flessner MF, Akylbekova EL, Fox CS, et al. The relation of C-reactive protein to chronic kidney disease in African Americans: the Jackson Heart Study. BMC Nephrol. 2010;11(1).

43. Shankar A, Sun L, Klein BE, Lee KE, Muntner P, Nieto FJ, Tsai MY, Cruickshanks KJ, Schubert CR, Brazy PC, et al. Markers of inflammation predict the long-term risk of developing chronic kidney disease: a population-based cohort study. Kidney Int. 2011;80(11):1231-8.

44. Remuzzi G, Cattaneo D, Perico N. The aggravating mechanisms of aldosterone on kidney fibrosis. J Am Soc Nephrol. 2008;19(8):1459-62.

45. Del Vecchio L, Procaccio M, Vigano S, Cusi D. Mechanisms of disease: the role of aldosterone in kidney damage and clinical benefits of its blockade. Nat Clin Pract Nephrol. 2007;3(1):42-9.

46. Hannemann A, Rettig R, Dittmann K, Volzke H, Endlich K, Nauck M, Wallaschofski $\mathrm{H}$. Aldosterone and glomerular filtration--observations in the general population. BMC Nephrol. 2014;15:44.

47. Stepien M, Stepien A, Wlazel RN, Paradowski M, Banach M, Rysz M, Rysz J. Obesity indices and adipokines in non-diabetic obese patients with early stages of chronic kidney disease. Med Sci Monit. 2013;19:1063-72

48. May A, Wang TJ. Biomarkers for cardiovascular disease: challenges and future directions. Trends Mol Med. 2008;14(6):261-7.

49. Cook NR. Use and misuse of the receiver operating characteristic curve in risk prediction. Circulation. 2007;115(7):928-35.

50. Wang TJ, Gona P, Larson MG, Tofler GH, Levy D, Newton-Cheh C, Jacques PF, Rifai N, Selhub J, Robins SJ, et al. Multiple biomarkers for the prediction of first major cardiovascular events and death. N Engl J Med. 2006;355(25):2631-9.

51. Melander $O$, Newton-Cheh C, Almgren P, Hedblad B, Berglund G, Engstrom G, Persson M, Smith JG, Magnusson M, Christensson A, et al. Novel and conventional biomarkers for prediction of incident cardiovascular events in the community. JAMA. 2009;302(1):49-57.

52. Kavousi M, Desai CS, Ayers C, Blumenthal RS, Budoff MJ, Mahabadi AA, Ikram MA, van der Lugt A, Hofman A, Erbel $R$, et al. Prevalence and prognostic implications of coronary artery calcification in low-risk women: a meta-analysis. JAMA. 2016;316(20):2126-34.

53. Zethelius B, Berglund L, Sundstrom J, Ingelsson E, Basu S, Larsson A, Venge $\mathrm{P}$, Arnlov J. Use of multiple biomarkers to improve the prediction of death from cardiovascular causes. N Engl J Med. 2008;358(20):2107-16.

54. Ridker PM, Buring JE, Rifai N, Cook NR. Development and validation of improved algorithms for the assessment of global cardiovascular risk in women: the Reynolds risk score. Jama. 2007;297(6):611-9.

55. Velagaleti RS, Gona P, Larson MG, Wang TJ, Levy D, Benjamin EJ, Selhub J, Jacques PF, Meigs JB, Tofler GH, et al. Multimarker approach for the prediction of heart failure incidence in the community. Circulation. 2010; 122(17):1700-6.
Ready to submit your research? Choose BMC and benefit from:

- fast, convenient online submission

- thorough peer review by experienced researchers in your field

- rapid publication on acceptance

- support for research data, including large and complex data types

- gold Open Access which fosters wider collaboration and increased citations

- maximum visibility for your research: over $100 \mathrm{M}$ website views per year

At BMC, research is always in progress.

Learn more biomedcentral.com/submissions 